\title{
A METHOD TO ESTIMATE WOOD SURFACE MOISTURE CONTENT DURING DRYING
}

\author{
G. Scheepers ${ }^{1, \star}$, P. Wiberg ${ }^{2}$, J. Johansson ${ }^{3}$
}

\begin{abstract}
A method to estimate the surface moisture content below the fibre saturation point that is a function of the surface temperature, wet- and dry bulb temperatures, equilibrium moisture content, and fibre saturation point was evaluated. The method is based on the premise that the surface temperature is solely influenced by the surface moisture content and the climate that the surface is exposed to. The prediction model contends that the surface moisture content is equal to the fibre saturation point when the surface temperature is equal to the wet bulb temperature, and equal to the equilibrium moisture content when the surface temperature is equal to the dry bulb temperature, with a linear interpolation between those two points. The model thus predicts that the average moisture content of a thin piece of veneer can be predicted with fairly good accuracy. Also, when drying boards in a fast changing climate, e.g. fan reversals in industrial kilns, the surface temperature and surface moisture content should change as abruptly as the climate does. Additionally, the surface moisture content should correlate to the known drying phases, with a consistently high surface moisture content during the capillary phase when the wet line is close to the surface, and a quickly decreasing surface moisture content when the wet line moves into the wood during the transition to the diffusion phase. The prediction model was tested in these three scenarios and the results suggest that the basic premise is reasonable, and that the method is useful for surface moisture content estimation.
\end{abstract}

Keywords: Dry bulb temperature, equilibrium moisture content, fibre saturation point, moisture measurement, surface temperature, wet bulb temperature.

\section{INTRODUCTION}

Proper control of wood surface moisture content is important during the drying of boards. If the wood surface becomes too dry at high internal moisture contents, it can lead to cracking and stresses in the boards. Even though the surface moisture content during drying is important to the final product quality, there has thus far not been a method to measure surface moisture content industrially. There have been academic examples of measuring surface moisture content measurement on small samples with near infrared (NIR), magnetic resonance and X-ray computed tomography scanning (Baettig et al. 2006, Rosenkilde and Glover 2002, Watanabe et al. 2011, Watanabe et al. 2013, Wiberg and Morén 1999). Electrical resistivity has also been used (Yamamoto et al. 2013). However, none of these methods are easily applied in the industrial environment and some also suffer from resolution 
problems, with NIR and medical X-ray methods measuring a layer of a millimetre or more in thickness.

The equilibrium moisture content of wood in different climates is known and more or less the same for different wood species. In a drying process, boards with a surface moisture content at the fibre saturation point or higher should have a surface temperature equal to the wet-bulb temperature due to the presence of free water, and a wood surface that is at the equilibrium moisture content should maintain a surface temperature equal to the dry bulb temperature since no evaporation would occur at the surface. As pointed out by Stamm (1967), even though water may be evaporated deep inside a board, only a lesser fraction would exit the board while staying in the gas phase and passing through pits. Most of the moisture would repeatedly be adsorbed and evaporated as it passes through cell walls and lumens on the way to the last cell wall at the surface. A small percentage may also migrate via the cell walls to the surface. Consequently, much water has to pass through the board surface cell walls and evaporate one last time, and thus the board surface cell walls should maintain a moisture content above the equilibrium moisture content as long as water is evaporating inside the board. While water is evaporating at the surface cell wall, the surface should maintain a temperature lower than the dry bulb temperature because of the heat needed for evaporation. The more water there is in the surface cell wall, the more evaporation occurs, and the lower the surface temperature would be.

Consequently, a possible method to estimate the surface moisture content could be based on the surface temperature, as measured by infrared methods, together with the wet- and dry bulb temperatures, equilibrium moisture content, and fibre saturation point. Such a prediction model would result in a surface moisture content estimation equal to the fibre saturation point when the surface temperature is equal to the wet bulb temperature, and a surface moisture content estimation equal to the equilibrium moisture content when the surface temperature is equal to the dry bulb temperature, with a linear interpolation between those two points.

The underlying assumption is thus that the surface temperature is solely influenced by the surface moisture content and the climate that the surface is exposed to. Consequently, it is assumed that the surface temperature is not influenced by the temperature of the material below the surface. The measurement method should however be sensitive to an exceedingly thin surface layer, and not obstruct evaporation or moisture flow. Hence, no contact measurement method would be acceptable, thus leaving infrared temperature measurement as the only viable option.

It is difficult, or impossible, to directly evaluate the proposed measurement technique, but the underlying theory does make a few measurable predictions. First, the average moisture content of a thin piece of veneer below 30\% moisture content can be measured with this method with fairly good accuracy since the core and surface would be in close proximity. Second, when drying boards in a fast changing climate, e.g. fan reversals in industrial kilns, the surface temperature and surface moisture content should change as abruptly as the dry bulb temperature since the temperature of the underlying material should not influence the surface temperature according to the theory. Third, the surface moisture content should correlate to the known drying phases, with a consistently high surface moisture content during the capillary phase when the wet line is close to the surface, and a quickly decreasing surface moisture content when the wet line moves into the wood during the transition to the diffusion phase. In this study we evaluate this surface moisture content measurement method in these three scenarios.

\section{MATERIALS AND METHODS}

Beech veneer pieces of $0,6 \times 70 \times 70 \mathrm{~mm}^{3}$ were soaked in water to saturation and dried at room temperature on a sensitive scale while the dry- and wet bulb temperatures were measured in close proximity with PT100 resistance thermometers and logged. The measured dry and wet bulb temperatures were typically $24,5^{\circ} \mathrm{C}$ and $17^{\circ} \mathrm{C}$, respectively. The veneer was supported to stand on edge, maximally exposing the veneer to the surrounding air and avoiding hindrances to evaporation on both flat sides. While the veneer was drying, its surface temperature was measured simultaneously by two Calex Electronics Ltd. PC21MT-X pyrometers with a $\pm 1^{\circ} \mathrm{C}$ accuracy and $\pm 0,5^{\circ} \mathrm{C}$ repeatability, 
fixed 0,95 emissivity setting, and spectral response in the 8-14 $\mu \mathrm{m}$ range. The measurement spots were approximately $30 \mathrm{~mm}$ in diameter in the centre of the veneer. The pyrometer readings were calibrated to the wet bulb temperature. After the veneer weight stabilised the experiment was terminated and the veneer oven dry weight was determined to be able to calculate the moisture content at each point in the drying process.

In a second investigation a $20 \mathrm{~mm}$ thick, $30 \mathrm{~mm}$ wide and $300 \mathrm{~mm}$ long end- and edge-sealed spruce (Picea abies) sapwood board was dried at $40^{\circ} \mathrm{C}$ while the surface temperature was measured with the two mentioned pyrometers at two different spots $200 \mathrm{~mm}$ from each other on a tangential surface of the board. The measurement spot diameters were about 2 to 3 centimeters in diameter and contained both earlywood and latewood. The sample was weighed throughout the drying process and the oven dry weight was determined at the end to calculate the average moisture content at every point in the drying process.

In a third investigation, the surface temperature of a stack of $50 \times 125 \mathrm{~mm}^{2}$ Scots Pine (Pinus sylvestris) of varying length in an industrial drying process was measured. The planks were sawn from 172-182 mm diameter logs. In this case the pyrometer was angled upwards and the measurement spot on the board stack was about 1 meter in diameter. The stack was outermost in the batch. The target moisture content was $18 \%$. The infrared temperature measurement was calibrated to the wet bulb temperature during a period in the beginning of the drying process when the measurement spot was on the leeward side and the difference between the dry and wet bulb temperatures was very small.

In each investigation the surface moisture content was estimated to be equal to the fibre saturation point when the surface temperature was equal to the wet bulb temperature, and equal to the equilibrium moisture content when the surface temperature was equal to the dry bulb temperature, with a linear interpolation between those two points, yielding the following equation:

$$
M_{s}=M_{e m c}+\frac{\left(T-T_{s}\right)}{\left(T-T_{w}\right)} x\left(M_{f s p}-M_{e m c}\right)
$$

for $T_{w} \leq T_{s} \leq T$

Where:

$M_{s}=$ surface moisture content (\%);

$M_{e m c}=$ equilibrium moisture content (\%);

$T=$ dry bulb temperature $\left({ }^{\circ} \mathrm{C}\right)$;

$T_{s}=$ surface temperature $(\%)$;

$T_{w}=$ wet bulb temperature $\left({ }^{\circ} \mathrm{C}\right)$; and

$M_{f s p}=$ fibre saturation point (\%), chosen as $30 \%$ moisture content in this study.

The intended use of the prediction model is for estimation of surface moisture contents where the surface temperature is between the dry and wet bulb temperatures, i.e. when water evaporates from the surface. For surface moisture content prediction during adsorption experimental data would first have to be collected on surface temperature development in this scenario. 


\section{RESULTS AND DISCUSSION}

The saturated Beech veneer had a starting moisture content of about $120 \%$. During drying down to about $30 \%$ moisture content (Figure 1), the prediction model gave a moisture content value of just beneath $30 \%$. Of course, the model can only predict moisture contents of $30 \%$ and below. Once the veneer moisture content fell below $30 \%$, the predicted values started to follow the measured moisture content. The root mean squared error of prediction (RMSEP) below $28 \%$ average moisture content was $2 \%$.

There were some factors that could have influenced these measurements negatively. One is the thickness of the veneer. Since the veneer was $0,6 \mathrm{~mm}$ thick it does not represent a true surface, where a surface can be described as a 2-dimensional structure, this was a 3 dimensional wood structure that was several cell layers thick. The square shaped veneer also dried faster at its four long edges than at the core, while the pyrometer was aimed at the core. This is probably the reason the pyrometer indicated a higher surface moisture content than the average moisture content after the average moisture content went below $30 \%$. Despite this the estimate followed the average moisture content closely enough to validate the prediction model.

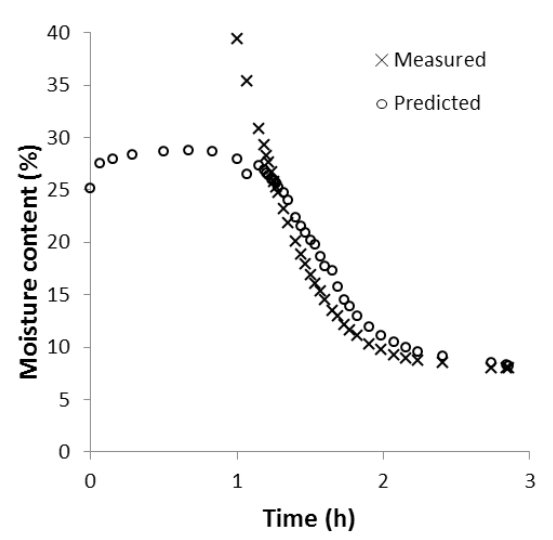

Figure 1. Measured and predicted moisture content values for 0,6 $\mathrm{mm}$ thick Beech veneer.

In previous wood drying research it has been shown that the wetline remains close to the surface for a large part of the drying process (Wiberg and Morén 1999). This phenomenon has been shown to occur in other wood species as well. So when measuring the surface moisture content of a board one would expect a fairly stable surface moisture content up to a certain point in the drying process when the surface would dry out rapidly.

In the next experiment the surface temperature was measured at two different spots, $c a .200 \mathrm{~mm}$ apart, on a spruce board with two different pyrometers. The predicted surface moisture content during drying is shown in Figure 2.

The predicted surface moisture content decreases progressively until $c a .40 \%$ average moisture content is reached. At this point the surface moisture content starts to decrease rapidly and linearly until about $30 \%$ average moisture content is reached, whereupon it bottoms out and slowly approaches the equilibrium moisture content. In a publication of Rémond et al. (2005) the surface moisture content of Norway spruce during drying was calculated by measuring thickness shrinkage. In this case, the surface moisture content also decreased rapidly from about $20 \%$ surface moisture content and $40 \%$ average moisture content, which was also the point where they estimated the wetline to recede. 


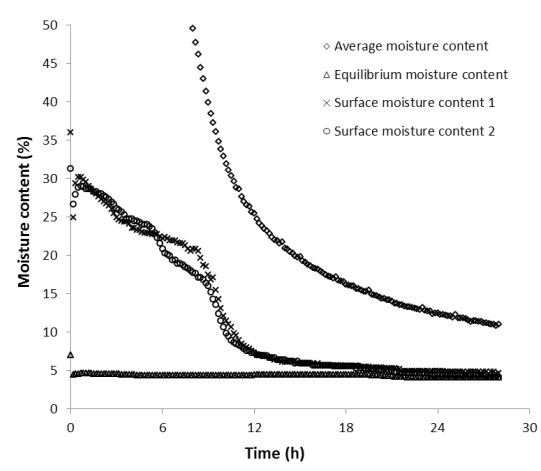

Figure 2. The surface moisture content development at two different measurement spots on a spruce plank during drying.

The nonlinear development of the surface moisture content to about $40 \%$ moisture content may be correlated to the nonlinear evolution of the capillary flow rate that can be expected at high moisture contents (Scheepers et al. 2007).

Figures 3 through 7 show the measurements in an industrial drying kiln. These measurements were done on one side of the drying batch, thus, with every fan reversal the measurement changed from being on the windward side to the leeward side, resulting in an abrupt climate change. The surface temperature measurement included both wetter sapwood and drier heartwood, but since only the board edges were visible, sapwood would dominate the temperature reading. The dry sticker ends probably also influenced the temperature readings slightly, though they accounted for a very small total area in the measurement spot. Nevertheless, the general surface moisture content development and other phenomena at different stages of drying were informative.

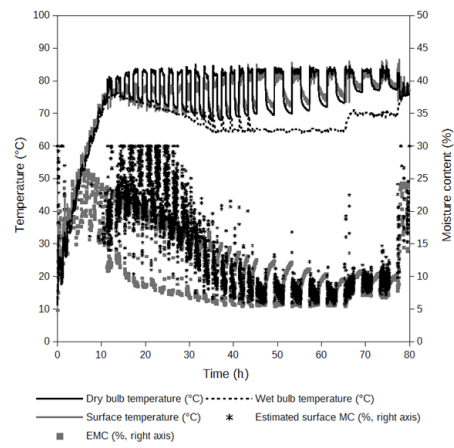

Figure 3. Data from an industrial kiln. The surface and equilibrium moisture contents are plotted against the right axis.

For surface temperature readings below the wet bulb temperature, a 30\% moisture content was estimated. If the surface temperature reading was above the dry bulb temperature, no surface moisture content was estimated since the model is not considered to apply to adsorption. 


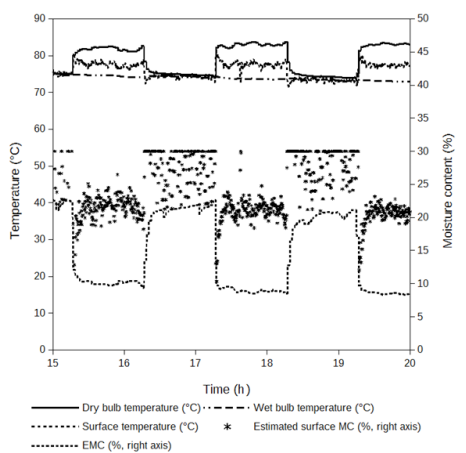

Figure 4. A snapshot from the early part of the drying run.

Figure 4 shows the temperatures, equilibrium and surface moisture contents at the start of the drying run. When the boards were on the windward side they had a surface moisture content of 20 to $25 \%$. When on the leeward side, the surface moisture content increased to 25 to $30 \%$, but still the equilibrium moisture content was lower than the surface moisture content, indicating that evaporation still occurred even though the dry- and wet bulb temperature difference was small. Thus, the surface moisture content increase while on the leeward side was due to moisture supply from the core of the lumber, and not due to net adsorption from the air.

The surface temperature reacted as abruptly as the dry bulb temperature when reversing the air flow direction, confirming the prediction model premise that the temperature of an exceedingly thin surface layer is independent of the temperature of the underlying material, while being exclusively dependent on the climate and surface moisture content.

An interesting result from Figure 4 is that the surface moisture content is close to the EMC at the start of a windward cycle. The surface moisture content then rises sharply over a number of minutes to eventually stabilise at a higher value. This phenomenon, if not a measurement artefact, suggests that it takes several minutes to set up a high stable moisture flux. Likewise, in Figure 5, it can be seen that the surface starts at a high moisture content when on the leeward side, and that the surface moisture content rapidly decreases over a number of minutes. If not a measurement artefact, it suggests that it takes several minutes to set up a slower moisture flux. In retrospect, it seems like this phenomenon is observable at the start of the spruce board experiment (Figure 2) as well.

Figure 5 shows a transitional period where the surface moisture content on the windward side starts to go below $20 \%$ moisture content. On the leeward side, the gap between the dry and wet bulb temperatures start to increase and the surface moisture content starts to decrease and rapidly approaches the surface moisture content on the windward side. Presumably, this is the point where the wetline starts to recede into the sapwood.

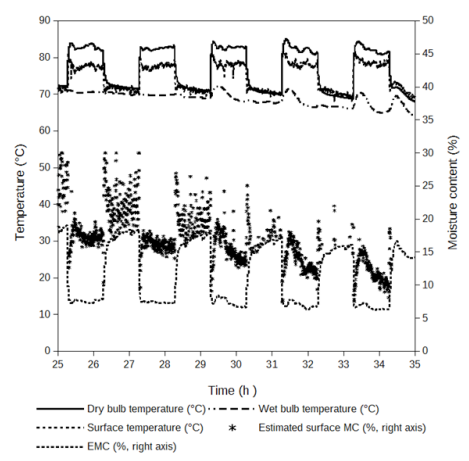

Figure 5. A snapshot from later in the drying run then when the surface moisture content starts to decrease rapidly. 
While the equilibrium moisture content on the leeward side is lower than the surface moisture content, drying of the surface occurs. At the point in the drying process when the surface moisture content on the leeward side is equal to the equilibrium moisture content, the surface stops drying when on the leeward side. Beyond this point the surface moisture content on the leeward side is below the equilibrium moisture content and the surface absorbs moisture from the air. Figure 6 also shows that the surface temperature on the leeward side towards the latter part of the drying process is higher than the dry bulb temperature. This is because latent heat is released when the water from the air is adsorbed onto the surface.

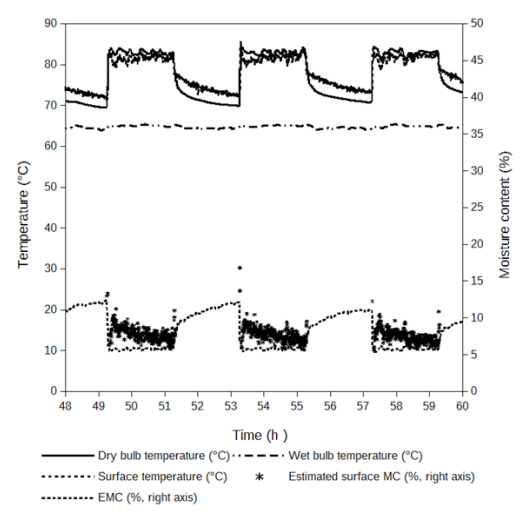

Figure 6. I snapshot toward the end of the drying run.

In Figure 6 the surface moisture content on the windward side at the point of fan reversal is about $7 \%$, while the equilibrium moisture content is about $5 \%$. When the fan direction is reversed, the equilibrium moisture content immediately goes up to about $8 \%$ after which it steadily increases to about $12 \%$, when the next reversal occurs. When the boards are on the windward side again the surface moisture content start at about $9 \%$, which means that the surface moisture content during the time when the boards were on the leeward side increased from $7 \%$ to $9 \%$. This increase was probably due to both adsorption from the air, and diffusion from the core of the lumber. Thus, the core of the lumber was drying and the surface moisture content increasing, decreasing the moisture content gradient.

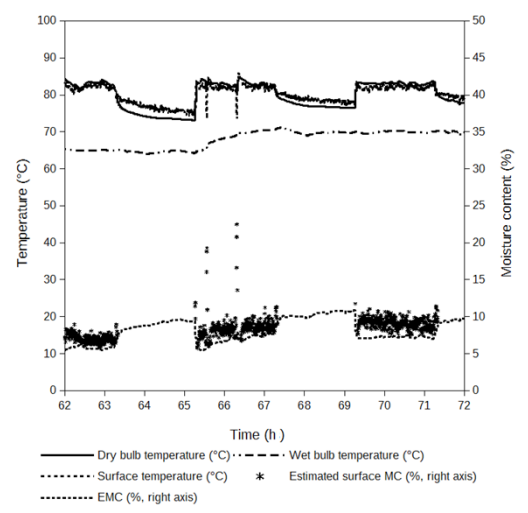

Figure 7. A snapshot of the very end of the drying run. After $c a$. 66 hours water spray droplets influenced the temperature measurements and surface moisture content estimation.

At the end of the drying run the wet bulb temperature was increased (Figure 7), resulting in a higher humidity climate that raised the surface moisture content to higher levels when on the windward side. 


\section{CONCLUSIONS}

The proposed surface moisture content measurement method is based on the premise that the surface temperature is solely influenced by the surface moisture content and the climate that the surface is exposed to. Hence, the model predicts that the average moisture content of a thin piece of veneer can be predicted with fairly good accuracy. Also, when drying boards in a fast changing climate, e.g. fan reversals in industrial kilns, the surface temperature and surface moisture content should change as abruptly as the dry bulb temperature. Additionally, the surface moisture content should correlate to the known drying phases, with a consistently high surface moisture content during the capillary phase when the wet line is close to the surface, and a quickly decreasing surface moisture content when the wet line moves into the wood during the transition to the diffusion phase.

The prediction model was tested in these three scenarios and the results confirm the associated predictions, thus suggesting that the basic premise is reasonable, and that the method is useful for surface moisture content estimation. The method is also applicable to other hygroscopic materials of which the saturation point and equilibrium moisture content in different climates is known.

\section{REFERENCES}

Baettig, R.; Rémond, R.; Perré, P. 2006. Measuring moisture content profiles in a board during drying: a polychromatic X-ray system interfaced with a vacuum/pressure laboratory kiln. Wood Science and Technology 40(4):261-274.

Rémond, R.; Perré, P.; Mougel, E. 2005. Using the concept of thin dry layer to explain the evolution of thickness, temperature, and moisture content during convective drying of Norway spruce boards. Drying technology 23(1-2):249-271.

Rosenkilde, A.; Glover, P. 2002. High resolution measurement of the surface layer moisture content during drying of wood using a novel magnetic resonance imaging technique. Holzforschung 56(3):312-317.

Scheepers, G.; Morén, T.; Rypstra, T. 2007. Liquid water flow in Pinus radiata during drying. Holz als Roh-und Werkstoff 65(4):275-283.

Stamm, A.J. 1967. Diffusion. Wood Science and Technology 1(3):205-230.

Watanabe, K.; Kobayashi, I.; Saito, S.; Kuroda, N.; Noshiro, S. 2013. Nondestructive evaluation of drying stress level on wood surface using near-infrared spectroscopy. Wood science and technology 47(2):299-315.

Watanabe, K.; Mansfield, S. D.; Avramidis, S. 2011. Application of near-infrared spectroscopy for moisture-based sorting of green hem-fir timber. Journal of wood science 57(4):288-294.

Wiberg, P.; Morén, T.J. 1999. Moisture flux determination in wood during drying above fibre saturation point using CT-scanning and digital image processing. European Journal of Wood and Wood Products 57(2):137-144.

Yamamoto, H.; Sakagami, H.; Kijidani, Y.; Matsumura, J. 2013. Dependence of microcrack behavior in wood on moisture content during drying. Advances in Materials Science and Engineering 2013: Article ID 802639, 7p. 